\title{
Genetic Determinations of Variable Responsiveness to Clopidogrel and Implications for Neurointerventional Procedures
}

\author{
Ruth Colley ${ }^{\mathrm{a}}$ Bernard Yan ${ }^{\mathrm{a}-\mathrm{c}}$ \\ ${ }^{a}$ Department of Medicine, University of Melbourne, ${ }^{b}$ Department of Neurology, and \\ 'Melbourne Brain Centre, Royal Melbourne Hospital, Parkville, Vic., Australia
}

\section{Key Words}

Clopidogrel hyporesponsiveness - CYP2C19 polymorphism • Stent thrombosis •

Neurointervention

\begin{abstract}
Endovascular intervention is emerging as a substitute for open surgical procedures for the treatment of cerebrovascular disease. However, up to $9 \%$ of patients undergoing neurointerventional procedures develop thromboembolic complications. Strategies to reduce periprocedural thromboembolic events are dominated by the use of dual antiplatelet therapy (DAT) which has been validated based on studies of peripheral vascular and coronary intervention. Of note, DAT decreases adverse vascular outcomes by $75-80 \%$ in patients undergoing percutaneous coronary intervention ( $\mathrm{PCl})$. It follows that similar treatment effects would be observed in neurointerventional populations. However, a growing body of evidence demonstrates that a subgroup of patients respond suboptimally to DAT, and in particular to clopidogrel (termed clopidogrel hyporesponders). These patients may be at an increased risk of thromboembolic complications such as in-stent thrombosis following neurointerventional procedures. Previous studies report 5-30\% suboptimal response to clopidogrel in the cardiovascular population, while a higher prevalence is seen in populations undergoing neurointerventional procedures, i.e. as much as $66 \%$. Knowledge of the mechanism leading to clopidogrel hyporesponsiveness is accumulating. A number of genetic polymorphisms, in particular CYP 2C19*2, have been associated with clopidogrel hyporesponsiveness and clinical outcomes. In addition, there are significant differences in the prevalence of CYP 2C19*2 across racial groups. Approximately $50 \%$ of Asians and $25 \%$ of Caucasians harbor the CYP 2C19*2 allele. While no prospective randomized
\end{abstract}


trials currently exist to demonstrate improved clinical outcomes with genotype-based treatment for carriers of the CYP 2 C $19 * 2$ polymorphism, a number of studies show that an increased dose of clopidogrel improves platelet inhibition in hyporesponders. The aim of the review is to examine the current understanding of the genetic basis of clopidogrel hyporesponsiveness in patients undergoing neurointerventional procedures and to explore current efforts using genotype and phenotype testing as well as alternative strategies to overcome the clopidogrel hyporesponsiveness.

Copyright $\odot 2012$ S. Karger AG, Base

\section{The Scope of Thromboembolic Complications in Neurointerventional Procedures}

Endovascular technological advancements have significantly improved the ability to treat cerebrovascular disease, in particular intracranial aneurysms and arterial stenoses. However, the transluminal placement of thrombogenic metallic devices (stents, flow diverting devices, coils) as part of neurointerventional procedures is associated with a risk of thromboembolic events [1]. Thromboembolic complications following coiling of aneurysms is estimated at up to 9.2\% [1] while carotid angioplasty or stent placement has been associated with ischemic complications in $3-13 \%$ of patients [2].

\section{Strategies to Decrease Thromboembolic Complications}

\section{Dual Antiplatelet Therapy in Coronary Intervention}

Trials of dual antiplatelet therapy (DAT) have confirmed the benefit of clopidogrel in addition to aspirin compared to aspirin monotherapy in prevention of death, myocardial infarction (MI), and stroke in cardiovascular patients undergoing percutaneous coronary intervention (PCI) [3]. A trial of more than 1,600 patients across 50 centers undergoing coronary stent placement found that DAT (325 mg aspirin daily plus $250 \mathrm{mg}$ ticlopidine twice daily) reduced the rate of stent thrombosis from $3.6 \%$ in the aspirin monotherapy group (325 mg once daily) to $0.6 \%$ in the DAT group [3]. Additionally, the authors reported a $75-80 \%$ relative risk reduction of cardiovascular death, MI, and stroke with the use of DAT.

\section{DAT in Neurointerventional Procedures}

To date, there are no randomized controlled trials in the neurointerventional patient population to demonstrate the benefits of DAT in reducing procedure-associated thromboembolic complications. Consequently, no well-established clinical guidelines exist to guide the use of DAT in this population. However, on the basis of randomized controlled trials in cardiology and expert opinion, the World Federation of Interventional and Therapeutic Neuroradiology (WFITN) has issued recommendations for DAT in the neurointervention setting. It is recommended that patients undergoing neurointerventional procedures receive aspirin $100 \mathrm{mg}$ and clopidogrel $75 \mathrm{mg}$ for 3 days prior to the procedure, with treatment duration to vary according to the nature of the intervention (www.wftin.org).

\section{Recurrent Ischemic Events Despite DAT}

Despite the obvious benefits of DAT in preventing thromboembolic complications after interventional procedures, there is a subpopulation of patients who develop recurrent ischemic events despite receiving therapeutic doses of antiplatelet agents. A review of trials in- 
vestigating the efficacy and safety of stent-assisted coiling as treatment for intracranial aneurysms reported that $6 \%$ of patients experienced clinically significant thromboembolic events while on DAT [4]. Authors of the CREST trial compared a variety of DAT regimens in patients undergoing carotid stenting or endarterectomy. Patients in the stenting arm had a rate of recurrent stroke or death of $6.8 \%$ at 4 years [5].

Increasing awareness of the subpopulation of patients continuing to suffer ischemia despite therapeutic DAT has led to significant research into the phenomenon of antiplatelet hyporesponsiveness. Patients diagnosed as aspirin and/or clopidogrel hyporesponders have a lower than expected biological response to a therapeutic dose of an antiplatelet drug.

\section{Variable Responsiveness to Clopidogrel}

Clopidogrel hyporesponsiveness is an increasingly recognized clinical phenomenon. Platelet responsiveness following a therapeutic dose of clopidogrel is suggested to follow a bell curve distribution [6]. A secondary post hoc analysis involving a variety of patient subgroups revealed a mean percent platelet inhibition of $41.9 \%$ following a standard dose of clopidogrel as measured by light transmission aggregometry [6]. Defining clopidogrel hyperor hyporesponsiveness as a platelet inhibition $2 \mathrm{SD}$ above or below the mean, respectively, patients with more than $45 \%$ or less than $37 \%$ platelet inhibition following a standard dose of clopidogrel were considered to be hyper- and hyporesponders, respectively [6]. Studies of cardiovascular patient populations suggest the prevalence of clopidogrel hyporesponsiveness to be between 5 and $30 \%$ [1] while current estimates indicate that the prevalence may be as high as $66 \%$ in patients undergoing neurointerventional procedures $[7,8]$. In part, variation in prevalence rates of clopidogrel hyporesponsiveness may be due to differing methodologies for its diagnoses.

\section{Clinical Implications of Clopidogrel Hyporesponsiveness}

Hyporesponsiveness to clopidogrel is translated into a significant clinical risk of ischemic events. Meta-analysis and other studies reveal hyporesponsiveness to clopidogrel as an independent risk factor associated with an increased risk of cardiac, cerebrovascular, and peripheral atherothrombotic events [9]. Further, it has recently been demonstrated that clopidogrel hyporesponders are at an increased risk of periprocedural stent thrombosis following interventional surgery. A study of 1,019 patients undergoing PCI and receiving a bare metal stent $(72.7 \%$ of study population) or drug-eluted stent (DES) (27.3\% of study population) found $32.3 \%$ to be hyporesponsive to clopidogrel $6 \mathrm{~h}$ after a 600 -mg loading dose as measured by light transmission aggregometry . At 3 months, $4.6 \%$ of clopidogrel hyporesponders had developed a stent thrombosis compared to $2 \%$ of responders (OR 2.31, 95\% CI 1.1-4.84, p = 0.02 ) [10]. These findings appear to hold true for patients undergoing neurointerventions [7, $11,12]$. A study of 53 Korean patients undergoing neurointerventional procedures revealed that $62.3 \%$ were hyporesponsive to clopidogrel 3 days after initiation of $75 \mathrm{mg} /$ day clopidogrel. Of note, 5 patients experienced thromboembolic complications after surgery, and all of them were hyporesponsive to clopidogrel with a mean percent platelet inhibition of $<23 \% \pm$ 2.3 [11]. To date, studies of clopidogrel hyporesponsiveness in the neurointervention patient population remain underpowered with small sample sizes. Research findings need to be replicated in larger cohorts to verify results.

Following increased awareness of the clinical risk associated with clopidogrel hyporesponsiveness, research attention is now focused on its potential causes. 


\section{Mechanisms of Clopidogrel Hyporesponsiveness}

Variation in platelet responsiveness to clopidogrel may arise from alterations in its pharmacokinetic or pharmacodynamic actions as a result of genetic or nongenetic influences. Factors such as age, body mass index (BMI), and comorbidities such as diabetes can influence the pharmacokinetic and dynamic response to clopidogrel [9]. It is important to recognize that clopidogrel hyporesponsiveness can be an expression of medication nonadherence and medication interaction. An example is the interaction with proton pump inhibitors involved in the pharmacokinetic and pharmacodynamic actions of clopidogrel, which may play a role in the development of hyporesponsiveness. Genes involved in clopidogrel absorption (ABCB1), biotransformation (CYP 1A2, CYP 2B6, CYP 2C19, CYP 2C9, CYP 3A4, and CYP 3A5), and pharmacodynamic response (P2Y12) have been investigated [13]. Genetic polymorphisms in these genes have been studied to varying degrees, with some appearing not to play a role in platelet responsiveness (P2Y12 and CYP 1A2 polymorphisms) while the role of other polymorphisms remains contentious (CYP 3A5, 3A4, 2B6 and 2C9) [13]. Polymorphisms of the ABCB1 gene appear to be associated with clopidogrel responsiveness compared with wild-type (genotype CC) carriers of 1 or 2 ABCB1 C3435T single-nucleotide polymorphisms have significantly reduced clopidogrel bioavailability following a 300- or 600 -mg loading dose and poorer cardiovascular outcomes at 1 year post-MI [13]. However, the most consistent genetic polymorphisms associated with clopidogrel responsiveness and adverse clinical outcomes are polymorphisms of the CYP 2C19 gene.

\section{Function and Prevalence of CYP 2 C19 Polymorphisms}

CYP 2C19 plays an important role in the biotransformation of clopidogrel. Clopidogrel is a thiopyridine antiplatelet agent requiring biotransformation into its active form by hepatic CYP isoenzymes. In the order of $85 \%$ of a standard dose of clopidogrel is inactivated via hepatic esterases, with the remaining 15\% converted into the active thiol metabolite. In a first step, the inactive prodrug is converted into an inactive intermediate metabolite and in a subsequent second step into its active thiol metabolite. CYP 2C19 is responsible for approximately $45 \%$ of first step and 20\% of second step reactions. Several polymorphisms of CYP 2C19 have been identified, each conferring a different level of enzyme activity. The CYP $2 \mathrm{C} 19^{*} 1$ polymorphism is considered to confer 'normal' enzyme activity while CYP $2 \mathrm{C} 19^{*} 2$ and *3 render a nonfunctional enzyme. CYP 2C19*2 and ${ }^{*} 3$ polymorphisms comprise 85 and $99 \%$ of loss of function alleles in Caucasian and Asian populations, respectively [14]. The CYP 2C19*2 allele has approximately twice the prevalence in Asian populations compared to Caucasians; approximately $50 \%$ of Chinese and $25 \%$ of Caucasians carry at least one copy of the polymorphism [15].

\section{CYP 2C19*2 Influences Response to Clopidogrel}

Genome-wide analysis has revealed that up to $12 \%$ of variation in platelet responsiveness is attributable to CYP $2 \mathrm{C} 19^{*} 2$ polymorphisms [16]. Cohort studies have demonstrated poorer platelet inhibition in carriers of the CYP $2 \mathrm{C} 19^{*} 2$ polymorphism compared to noncarriers [17]. A genetic dose effect is seen whereby poor CYP 2 C19*2 metabolizers $\left({ }^{*} 2 / * 2\right.$ homozygotes) have poorer platelet inhibition compared to intermediate CYP 2C19*2 metabolizers (*1/*2 heterozygotes) [18]. Genetic analysis of 162 healthy volunteers demonstrated intermediate metabolizers and poor metabolizers to have $26-31 \%$ and $46-55 \%$ lower clopidogrel metabolite exposure, respectively, compared to noncarriers [19]. 


\section{CYP 2C19*2 Influences Clinical Outcomes}

In patients with cardiovascular disease but without having received endovascular treatment, there is little influence of the CYP $2 \mathrm{C} 19^{*} 2$ genotype on the clinical outcome [20]. Parè et al. [20] performed a genetic subanalysis on 2,549 patients randomly assigned clopidogrel and 2,510 assigned placebo (both in combination with aspirin) who were enrolled into the CURE or ACTIVE A trial. It was determined that clopidogrel addition to aspirin was superior to placebo in preventing recurrent ischemic events and Parè et al. [20] concluded that this benefit was not attenuated by the CYP 2C19 genotype. However, these results are in contrast to reports in patient populations undergoing PCI. Mega et al. [19] carried out a genetic substudy of 1,477 patients enrolled into the TRITON-TIMI 38. They reported carriage of the CYP $2 \mathrm{C} 19^{*} 2$ polymorphism to be associated with a $53 \%$ increase in poor cardiovascular outcomes, and in the subgroup undergoing PCI a 3-fold increase in stent thrombosis compared to noncarriers (2.6 vs. $0.8 \%$, HR for carriers $=3.09,95 \%$ CI $1.19-8, \mathrm{p}=0.02$ ) was found [19]. These findings are reflective of a number of cohort and case-control studies suggesting that carriage of the CYP $2 \mathrm{C} 19^{*} 2$ polymorphism is associated with an increased risk of ischemic events, including stent thrombosis, in clopidogreltreated patients undergoing PCI [21-23]. In addition, a meta-analysis of 9 trials evaluating the CYP 2C19 genotype on clinical outcomes in clopidogrel-treated patients revealed an increased risk of stent thrombosis in carriers of both 1 (HR 2.67; 95\% CI 1.69-4.22; p < $0.0001)$ and 2 (HR 3.97; 95\% CI 1.75-9.02; $\mathrm{p}=0.001)$ CYP 2C19*2 polymorphisms compared to noncarriers [24].

Recent studies have focused on the clinical outcomes of Asian patients who carry CYP 2C19*2 polymorphisms. Following 1,738 Chinese patients with coronary artery disease (CAD) and undergoing PCI, Luo et al. [25] found carriage of the CYP 2C19*2 polymorphism to be an independent predictor of stent thrombosis (HR for carriers $=4.26,95 \% \mathrm{CI}$ $1.28-9.22, \mathrm{p}<0.05)$. A small study of Japanese patients $(\mathrm{n}=100)$ receiving a DES found the incidence of stent thrombosis but not of major adverse cardiac events to be significantly higher in carriers of the CYP $2 \mathrm{C}^{*} 9^{*}$ polymorphism compared to noncarriers (52.3 vs. $15.5 \%, \mathrm{p}=0.0002)$ [26].

To our knowledge, no studies have associated CYP 2C19*2 polymorphisms and clinical outcomes in a patient population undergoing neurointerventional procedures. However, it follows that, in clopidogrel-treated patients, carriers of the CYP $2 \mathrm{C} 19^{*} 2$ polymorphisms may be at a heightened risk of stent thrombosis following neurointerventional procedures.

\section{The Food and Drug Administration Clopidogrel Black Box Warning}

In response to the evidence of reduced efficacy of clopidogrel in patients with CYP $2 \mathrm{C} 19^{*} 2$ polymorphisms, the US Food and Drug Administration (FDA) issued a boxed warning in March 2010 recommending physicians consider alternative management strategies in patients with the poor metabolizer genotype ( ${ }^{*} 2{ }^{*} 2$ homozygotes) (www.fda.gov). Controversially, the warning did not make note of the evidence suggesting that intermediate metabolizers $\left({ }^{*} 1 /{ }^{*} 2\right.$ heterozygotes) may also have reduced clopidogrel efficacy. The warning focused on a crossover study of 40 healthy volunteers, which demonstrated decreased metabolite exposure and increased platelet aggregation in poor metabolizers following both standard and double dose clopidogrel regimens. 


\section{The Role of CYP 2C19 Genotype Testing}

Given the evidence supporting the impact of CYP $2 \mathrm{C} 19^{*} 2$ on clopidogrel responsiveness and clinical outcomes, we believe that it is reasonable to recommend the genotype testing in patients planned for neurointervention procedures. In the following section, we will outline the different management strategies.

\section{Management Strategies for Patients with the CYP 2C19*2 Polymorphism}

\section{Increased Dosage of Clopidogrel?}

There is evidence that improved platelet inhibition could be achieved by increased doses of clopidogrel in patients with CYP 2C19*2 demonstrating clopidogrel hyporesponsiveness. Fontana et al. [27] measured platelet function in 81 patients who had recently undergone PCI and had been taking $75 \mathrm{mg}$ clopidogrel daily for 15 days. The patients diagnosed as hyporesponders (55.6\%) were changed to $150 \mathrm{mg} /$ day clopidogrel and experienced a decrease in mean platelet reactivity from 62 to $49.4 \%$ after 15 days ( $p<0.001$ ). Furthermore, results from the PRINC trial examined the effect of differing clopidogrel regimens on platelet inhibition in patients undergoing PCI [28]. A second 600-mg loading dose $2 \mathrm{~h}$ after the first produced better acute platelet inhibition ( $42 \%$ inhibition with clopidogrel vs. $24 \%$ with placebo). The trial also demonstrated that an increased maintenance dose of $150 \mathrm{mg}$ produced superior platelet inhibition after 1 week compared to $75 \mathrm{mg}$ in clopidogrel hyporesponders [28].

However, it remains to be demonstrated that increased doses of clopidogrel achieve superior platelet inhibition and improved clinical outcomes in carriers of the CYP $2 \mathrm{C} 19^{*} 2$ polymorphism.

\section{Alternatives to Clopidogrel}

Alternative thiopyridine agents such as prasugrel and ticagrelor have been considered in patients with poor response to clopidogrel. Prasugrel, requiring reduced hepatic conversion in comparison to clopidogrel, appears to have few hyporesponders and produces superior platelet inhibition in comparison to 75 or $150 \mathrm{mg}$ clopidogrel [29]. However, it is noted that prasugrel has an increased bleeding risk compared to clopidogrel and is therefore unlikely to be an appropriate alternative in patients with ischemic stroke [14]. Ticagrelor, although not yet approved for clinical use, does not require hepatic biotransformation. It is seen to be effective in reducing the risk of vascular death, MI, stroke, and stent thrombosis without an increased bleeding risk [14]. Furthermore, ticagrelor appears to be effective in improving platelet inhibition in clopidogrel hyporesponders [30]. Another alternative management strategy under investigation is the addition of a third antiplatelet agent to aspirin and clopidogrel in patients who are seen to be hyporesponsive on tests of platelet function. Addition of cilostazol (100 mg twice daily) to standard clopidogrel and aspirin therapy in patients with clopidogrel hyporesponsiveness provided a greater degree of platelet inhibition compared to high dose clopidogrel in hyporesponsive patients undergoing PCI [14].

However, both prasugrel and ticagrelor are yet to be validated as superior therapies in CYP 2 C19*2 poor and intermediate metabolizers. 


\section{Ongoing Clinical Trials}

Currently, there are no prospective trials demonstrating a superior benefit of targeted treatment for patients undergoing interventional procedures based on the CYP 2C19 genotype. The GeCCO (genotype-guided comparison of clopidogrel and prasugrel outcomes study) is the largest open-label cohort study planned to investigate the efficacy of a genotypeguided comparison of therapies based on the CYP 2C19 genotype [14]. The study aims to recruit 14,600 subjects and compare the outcomes of patients assigned to $75 \mathrm{mg}$ clopidogrel or 5-10 mg prasugrel based on genotype. The ACCEL-2C19 study plans to randomly assign 80 patients with stable CAD undergoing PCI to receive high dose clopidogrel (150 mg) plus $100 \mathrm{mg}$ aspirin or cilostazol $100 \mathrm{mg}$ twice daily based on the CYP 2C19 genotype [14]. Additionally, they will measure platelet responsiveness at 30 days.

Platelet function testing is another means of guiding therapy. In contrast to genotypebased assays, platelet function assays are available as convenient point-of-care devices; however, they lack specificity. The largest trial currently examining the efficacy of a phenotypeguided comparison of therapies based on platelet responsiveness to clopidogrel is GRAVITAS (gauging responsiveness with a verify now assay - impact on thrombosis and safety) [14]. GRAVITAS aims to recruit 2,800 patients with stable CAD or acute coronary syndrome undergoing PCI with DES placement. Patients with poor platelet inhibition 12-24 h after DES insertion will be randomized to a standard $75 \mathrm{mg}$ or a high dose clopidogrel regimen (an additional 600-mg loading dose followed by $150 \mathrm{mg}$ daily). A substudy of GRAVITAS, GIFT, will aim to assess the association between CYP 2C19 genotype and platelet responsiveness.

\section{Conclusion}

A significant proportion of patients undergoing neurointerventional procedures are hyporesponsive to clopidogrel. Carriage of the CYP 2C19*2 allele is significantly associated with clopidogrel hyporesponsiveness and poses an increased risk of ischemic events, including stent thrombosis, following PCI. Although these findings remain to be verified in neurointerventional patient populations, CYP 2C19*2 screening may form part of neurointerventional practice in the future. Management strategies such as an increased dose of clopidogrel and alternative antiplatelet agents hold promise as viable alternative in patients with clopidogrel hyporesponsiveness but require validation from ongoing trials and in patients with the CYP 2C19*2 allele.

\section{Disclosure Statement}

R.C. and B.Y. affirm no conflicts of interest. 


\section{References}

1 Oxley TJ, Dowling RJ, Mitchell PJ, Davis SM, Yan B: Antiplatelet resistance and thromboembolic complications in neurointerventional procedures. Front Neurol 2011;2:1-9.

-2 Qureshi AI, Suri MF, Ali Z, Kim SH, Lanzino G, Fessler RD, Ringer AJ, Guterman LR, Hopkins LN: Carotid angioplasty and stent placement: a prospective analysis of perioperative complications and impact of intravenously administered abciximab. Neurosurgery 2002;50:466-473.

-3 Leon MB, Baim DS, Popma JJ, Gordon PC, Cutlip DE, Ho KK, Giambartolomei A, Diver DJ, Lasorda DM, Willians DO, Pocock SJ, Kuntz for the Stent Anticoagulation Restenosis Study Investigators: A clinical trial comparing three antithrombotic-drug regimens after coronary-artery stenting. N Engl J Med 1998;339:1665.

4 Bodily KD, Cloft HJ, Lanzino G, Fiorella DJ, White PM, Kallmes DF: Stent-assisted coiling in acutely ruptures intracranial aneurysms: a qualitative, systematic review of the literature. AJNR Am J Neuroradiol 2011;32:1232-1236.

-5 Brott TM, Hobson RM, Howard GD, Roubin GM, Clark WM, Brooks WM, Mackey AM, Hill MM, Leimgruber PM, Sheffet AP, Howard VP, Moore WM, Voeks JP, Hopkins LM, Cutlip DM, Cohen DM, Popma JM, Ferguson RM, Cohen SM, Blackshear JM, Silver FM, Mohr JM, Lal BM, Meschia JM: Stenting versus endarterectomy for treatment of carotid-artery stenosis. N Engl J Med 2010;363:11-23.

-6 Serebruany VL, Steinhubl SR, Beger PB, Malinin AL, Bhatt DL, Topol EJ: Variability in platelet responsiveness to clopidogrel among 544 individuals. J Am Coll Cardiol 2005;45:246-251.

7 Lee DH, Arat A, Morsi H, Shaltoni H, Harris JR, Mawad ME: Dual antiplatelet therapy monitoring for neurointerventional procedures using a point-of-care platelet function test: a single-center experience. AJNR Am J Neuroradiol 2008;29:1389-1394.

8 Pandya DJ, Fitzsimmons BF, Wolfe TJ, Hussain SI, Lynch JR, Ortega-Gutierrez S, Zaidat OO: Measurement of antiplatelet inhibition during neurointerventional procedures: the effect of antithrombotic duration and loading dose. J Neuroimaging 2010;20:64-69.

-9 Sofi F, Marcucci R, Gori AM, Giusti B, Abbate R, Gensini GF: Clopidogrel non-responsiveness and risk of cardiovascular morbidity: an updated meta-analysis. Thromb Haemost 2010;103:841-848.

-10 Geisler T, Zürn C, Simonenko R, Rapin M, Kraibooj H, Kilias A, Bigalke B, Stellos K, Schwab M, May AE, Herdeg C, Gawaz M: Early but not late stent thrombosis is influenced by residual platelet aggregation in patients undergoing coronary interventions. Eur Heart J 2010;31:59-66.

-11 Ryu DS, Hong CK, Sim YS, Kim CH, Jung JY, Joo JY: Anti-platelet drug resistance in the prediction of thromboembolic complications after neurointervention. J Korean Neurosurg Soc 2010;48:319-324.

12 Rho GJ, Shin WR, Kong TS, Kim MS, Lee CJ, Lee BH: Significance of clopidogrel resistance related to the stent-assisted angioplasty in patients with atherosclerotic cerebrovascular disease. J Korean Neurosurg Soc 2011;50:40-44. Huber K: Genetic variability in response to clopidogrel therapy: clinical implications. Eur Heart J 2010;31:2974-2976. Society for Cardiovascular Angiography and Intervention, Society of Thoracic Surgeons, Writing Committee Members, Holmes DR, Dehmer GJ, Kaul S, Leifer D, O’Gara PT, Stein CM: ACCF/AHA clopidogrel clinical alert: approaches to the FDA 'boxed warning' - a report of the American College of Cardiology Foundation Task Force on Clinical Expert Consensus Documents and the American Heart Association. Circulation 2010;122:537-557.

15 Man M, Farmen M, Dumaual C, Teng CH, Moser B, Irie S, Noh GJ, Njau R, Close S, Wise S, Hockett R: Genetic variation in metabolizing enzyme and transporter genes. J Clin Pharmacol 2010;50:929-940.

-16 Shuldiner AR, O’Connell JR, Bliden KP, Gandhi A, Ryan K, Horenstein RB, Damcott CM, Pakyz R, Tantry US, Gibson Q, Pollin TI, Post W, Parsa A, Mitchell BD, Faraday N, Herzog W, Gurbel PA: Association of cytochrome P450 2C19 genotype with the antiplatelet effect and clinical efficacy of clopidogrel therapy. JAMA 2009;302:849-857.

- 17 Holmes MV, Perel P, Shah T, Hingorani AD, Casas JP: CYP2C19 genotype, clopidogrel metabolism, platelet function, and cardiovascular events: a systematic review and meta-analysis. JAMA 2011;306:2704-2714.

- 18 Maeda A, Ando H, Asai T, Ishiguro H, Umemoto N, Ohta M, Morishima M, Sumida A, Kobayashi T, Hosohata K, Ushijima K, Fujimura A: Differential impacts of CYP2C19 gene polymorphisms on the antiplatelet effects of clopidogrel and ticlopidine. Clin Pharmacol Ther 2011;89:229-233.

19 Mega JL, Close SL, Wiviott SD, Shen L, Hockett RD, Brandt JT, Walker JR, Antman EM, Macias W, Braunwald E, Sabatine MS: Cytochrome P450 polymorphisms and response to clopidogrel. N Engl J Med 2009;360:354-362.

20 Paré G, Mehta SR, Yusuf S, Anand SS, Connolly SJ, Hirsh J, Simonsen K, Bhatt DL, Fox KA, Eikelboom JW: Effects of CYP2C19 genotype on outcomes of clopidogrel treatment. N Engl J Med 2010;363:1704-1714.

-21 Giusti B, Gori AM, Marcucci R, Saracini C, Sestini I, Paniccia R, Buonamici P, Antoniucci D, Abbate R, Gensini GF: Relation of cytochrome P450 2C19 loss-of-function polymorphism to occurrence of drug-eluting coronary stent thrombosis. Am J Cardiol 2009;103:806-811.

22 Harmsze AM, van Werkum JW, ten Berg JM, Zwart B, Bouman HJ, Breet NJ, van 't Hof AW, Ruven HJ, Hackeng CM, Klungel OH, de Boer A, Deneer VH: CYP2C19*2 and CYP2C9*3 alleles are associated with stent thrombosis: a casecontrol study. Eur Heart J 2010;31:3046-3053.

23 Sibbing D, Stegherr J, Latz W, Koch W, Mehilli J, Dörrler K, Morath T, Schömig A, Kastrati A, von Beckerath N: Cytochrome P450 2C19 loss-of-function polymorphism and stent thrombosis following percutaneous coronary intervention. Eur Heart J 2009;30:916-922. 
24 Mega JL, Simon T, Collet JP, Anderson JL, Antman EM, Bliden K, Cannon CP, Danchin N, Giusti B, Gurbel P, Horne BD, JS H, Kastrati A, Montalescot G, Neumann FJ, Shen L, Sibbing D, Steg PG, Trenk D, Wiviott SD, Sabatine MS: Reduced-function CYP2C19 genotype and risk of adverse clinical outcomes among patients treated with clopidogrel predominantly for PCI: a meta-analysis. JAMA 2010;304:1821-1830.

25 Luo Y, Zhao YT, Verdo A, Qi WG, Zhang DF, Hu B: Relationship between cytochrome P450 2C19*2 polymorphism and stent thrombosis following percutaneous coronary intervention in Chinese patients receiving clopidogrel. J Int Med Res 2011;39:2012-2019.

26 Sawada T, Shinke T, Shite J, Honjo T, Haraguchi Y, Nishio R, Masakazu Shinohara M, Toh R, Ishida T, Kawamori H, Kozuki A, Inoue T, Hariki H, Hirata KI: Impact of cytochrome P50 2C19*2 polymorphism on intra-stent thrombus after drug-eluting stent implantation in Japanese patients receiving clopidogrel. Circ J 2011;75:99-105.

27 Fontana P, Senouf D, Mach F: Biological effect of increased maintenance dose of clopidogrel in cardiovascular outpatients and influence of the cytochrome P450 2C19*2 allele on clopidogrel responsiveness. Thromb Res 2008;121: 463-468.

28 Gladding P, Webster M, Zeng I, Farrell H, Stewart J, Ruygrok P, Ormiston J, El-Jack S, Armstrong G, Kay P, Scott D, Gunes A, Dahl ML: The antiplatelet effect of higher loading and maintenance dose regimens of clopidogrel: the PRINC (Plavix Response in Coronary Intervention) Trial. JACC Cardiovasc Interv 2008;1:612-619.

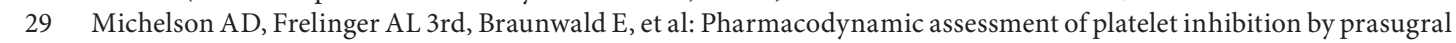
vs. clopidogrel in the TRITON-TIMI 38 trial. Eur Heart J 2009;30:1753-1763.

- 30 Gurbel PA, Bliden KP, Butler K, Antonino MJ, Wei C, Teng R, Rasmussen L, Storey RF, Nielsen T, Eikelboom JW, Sabe-Affaki G, Husted S, Kereiakes DJ, Henderson D, Patel DV, Tantry US: Response to ticagrelor in clopidogrel nonresponders and responders and effect of switching therapies: the RESPOND study. Circulation 2010;121:1188-1199. 